\title{
Topology and magnetism in the Kondo insulator phase diagram
}

\author{
Klett, Michael ; Ok, Seulgi ; Riegler, David ; Wölfle, Peter ; Thomale, Ronny ; Neupert, Titus
}

\begin{abstract}
Topological Kondo insulators are a rare example of an interaction-enabled topological phase of matter in three-dimensional crystals - making them an intriguing but also hard case for theoretical studies. Here, we aim to advance our theoretical understanding by solving the paradigmatic two-band model for topological Kondo insulators using a fully spin-rotation invariant slave-boson treatment. Within a meanfield approximation, we map out the magnetic phase diagram and characterize both antiferromagnetic and paramagnetic phases by their topological properties. Among others, we identify an antiferromagnetic insulator that shows, for suitable crystal terminations, topologically protected hinge modes. Furthermore, Gaussian fluctuations of the slave-boson fields around their mean-field value are included in order to establish the stability of the mean-field solution through computation of the full dynamical susceptibility.
\end{abstract}

DOI: https://doi.org/10.1103/physrevb.101.161112

Posted at the Zurich Open Repository and Archive, University of Zurich

ZORA URL: https://doi.org/10.5167/uzh-187547

Journal Article

Published Version

Originally published at:

Klett, Michael; Ok, Seulgi; Riegler, David; Wölfle, Peter; Thomale, Ronny; Neupert, Titus (2020). Topology and magnetism in the Kondo insulator phase diagram. Physical review B, 101(16):161112.

DOI: https://doi.org/10.1103/physrevb.101.161112 


\title{
Topology and magnetism in the Kondo insulator phase diagram
}

\author{
Michael Klett $\odot,{ }^{1}$ Seulgi Ok, ${ }^{2}$ David Riegler $\odot,{ }^{1}$ Peter Wölfle $\odot,{ }^{3}$ Ronny Thomale $\odot,{ }^{1,{ }^{*}}$ and Titus Neupert $\odot^{2, \dagger}$ \\ ${ }^{1}$ Institute for Theoretical Physics and Astrophysics, University of Würzburg, Am Hubland, D-97074 Würzburg, Germany \\ ${ }^{2}$ Department of Physics, University of Zurich, Winterthurerstrasse 190, 8057 Zurich, Switzerland \\ ${ }^{3}$ Institute for Theory of Condensed Matter, Karlsruhe Institute of Technology, 76131 Karlsruhe, Germany
}

(Received 5 December 2019; revised manuscript received 17 February 2020; accepted 24 March 2020; published 21 April 2020)

\begin{abstract}
Topological Kondo insulators are a rare example of an interaction-enabled topological phase of matter in three-dimensional crystals - making them an intriguing but also hard case for theoretical studies. Here, we aim to advance our theoretical understanding by solving the paradigmatic two-band model for topological Kondo insulators using a fully spin-rotation invariant slave-boson treatment. Within a mean-field approximation, we map out the magnetic phase diagram and characterize both antiferromagnetic and paramagnetic phases by their topological properties. Among others, we identify an antiferromagnetic insulator that shows, for suitable crystal terminations, topologically protected hinge modes. Furthermore, Gaussian fluctuations of the slave-boson fields around their mean-field value are included in order to establish the stability of the mean-field solution through computation of the full dynamical susceptibility.
\end{abstract}

DOI: 10.1103/PhysRevB.101.161112

Introduction. Landau's theory of spontaneous symmetry breaking and topological phenomena are often cited as two antipodal concepts by which phases of matter can be organized. However, in strongly correlated topological systems, which are surprisingly rare in three-dimensional systems, they can show an intriguing interplay. One of the paradigmatic examples are topological Kondo insulators (TKIs) [1,2], in which $d$ and $f$ electron bands partially overlap and hybridize. This band overlap sources the band inversion central to topological band theory, while correlations from the strongly localized $f$ electrons induce a robust hybridization gap between these bands and thus bring about an insulating ground state.

Several materials fall in this category, with $\mathrm{SmB}_{6}$ being the best-studied example. The nature of its ground state is still under debate despite intense experimental investigations, with evidence for a topological [3-8] as well as a nontopological [9] scenario, while some works even report indications for a metallic state [10-12]. Another point of controversy is the magnetic order of $\mathrm{SmB}_{6}$, with indications for paramagnetic (PM) [13], A-type antiferromagentic (AFM) [8,14], NeelAFM $[9,15,16]$, and surface-ferromagnetic phases. The presence of magnetism would crucially influence the topological classification of the material. Jointly, these results demonstrate that $\mathrm{SmB}_{6}$ and, more broadly, TKIs are at a nontrivial intersection of topology, symmetry-breaking, and correlated phenomena.

This renders the systems not only as highly relevant but also an intrinsically hard case for theoretical studies. Analytical and numerical methods are challenged by a strongly correlated interplay between localized and delocalized as well as spin and orbital degrees of freedom. In this work, we adopt

\footnotetext{
*rthomale@physik.uni-wuerzburg.de

†titus.neupert@uzh.ch
}

Kotliar-Ruckenstein's formulation of the slave-boson formalism [17], which matches with the Gutzwiller approximation in infinite dimensions, to map out its magnetic and topological phase diagram. We use Kotliar and Ruckenstein's scheme among the many variants of slave-boson formulations because it has been extended to a spin-rotation invariant description $[18,19]$, including Gaussian fluctuations [20-22], and refer to it shortly as slave bosons from here on.

In the slave-boson treatment, one replaces the fermionic creation and annihilation operators by a new set of fermionic ones combined with an auxiliary bosonic field. The bosonic fields are chosen such that the fermionic interaction terms are replaced by quadratic bosonic terms in the action, while the resulting pseudofermionic fields also only enter quadratically. When local constraints are imposed through Lagrange multiplier fields to constrain the values of bosonic fields to physical ones, one obtains an exact representation of the original problem. We employ a mean-field approximation for the bosonic fields, which imposes the constraints on average and allows for magnetic and nonmagnetic solutions. Following Ref. [22], one can acquire the expressions with the physical information of the original system, e.g., effective mass, spin, and charge susceptibility. Together with the periodic Anderson model, Kondo systems have been considered as a particularly suitable target to prove the accuracy of slave bosons for their physics including interacting electrons as well as hybridized orbitals.

In this work, we numerically implement the analytical representations from the slave-boson formalism and present the phase diagram for a model that resembles the low-energy physics in $\mathrm{SmB}_{6}$, but can be seen as paradigmatic for generic three-dimensional Kondo models with cubic and time-reversal symmetry. We find a total of seven phases, which are magnetically or topologically distinct, by tuning the strength of the electron-electron interactions as well as the on-site energy of the $f$ electrons. Besides various PM insulating topological 
phases, we find two topologically distinct insulating phases with $(\pi, \pi, \pi)$ (Neel-)AFM order.

Model and method. We start with a short exposition of our model and the main ingredients of the spin-rotation invariant slave-boson formalism. The Hamiltonian introduced in Ref. [23] as a minimal model for $\mathrm{SmB}_{6}$ reads

$$
\begin{gathered}
H=H_{0}+H_{\mathrm{hyb}}+H_{\mathrm{int}}, \\
H_{0}=\sum_{i} \tilde{\boldsymbol{f}}_{i}^{\dagger} \epsilon_{f} \widetilde{\boldsymbol{f}}_{i}-\sum_{\langle i, j\rangle}\left(\tilde{\boldsymbol{f}}_{i}^{\dagger} t^{f} \widetilde{\boldsymbol{f}}_{j}+\boldsymbol{c}_{i}^{\dagger} t^{d} \boldsymbol{c}_{j}+\text { H.c. }\right) \\
-\sum_{\langle\langle i, j\rangle\rangle}\left(\tilde{\boldsymbol{f}}_{i}^{\dagger} t^{f^{\prime}} \tilde{\boldsymbol{f}}_{j}+\boldsymbol{c}_{i}^{\dagger} t^{d^{\prime}} \boldsymbol{c}_{j}+\text { H.c. }\right)-\sum_{i} \mu_{0} \tilde{n}_{i}, \\
H_{\mathrm{hyb}}=\sum_{\alpha} \sum_{\langle i, j\rangle_{\alpha}} i V\left(\tilde{\boldsymbol{f}}_{i}^{\dagger} \tau^{\alpha} \boldsymbol{c}_{j}+\boldsymbol{c}_{i}^{\dagger} \tau^{\alpha} \tilde{\boldsymbol{f}}_{j}+\text { H.c. }\right), \\
H_{\mathrm{int}}=U \sum_{i} \tilde{f}_{i, \uparrow}^{\dagger} \tilde{f}_{i, \uparrow} \tilde{f}_{i, \downarrow}^{\dagger} \tilde{f}_{i, \downarrow}
\end{gathered}
$$

representing $f$ - and $d$-electron hopping on a simple cubic lattice, their hybridization, and the local repulsive Hubbard interaction, respectively. The spinors $\tilde{\boldsymbol{f}}_{i}^{\dagger}:=\left(\tilde{f}_{i, \uparrow}^{\dagger}, \tilde{f}_{i, \downarrow}^{\dagger}\right)$ and $\boldsymbol{c}_{i}^{\dagger}=\left(c_{i, \uparrow}^{\dagger}, c_{i, \downarrow}^{\dagger}\right)$ are formed by the creation operators of $d$ - $(f-)$ electrons $c_{i, \sigma}^{\dagger}\left(\tilde{f}_{i, \sigma}^{\dagger}\right)$ with spin $\sigma \in\{\uparrow, \downarrow\}$, and $\tilde{n}_{i}=\tilde{\boldsymbol{f}}_{i}^{\dagger} \widetilde{\boldsymbol{f}}_{i}+$ $\boldsymbol{c}_{i}^{\dagger} \boldsymbol{c}_{i}$ represents the local density of all electrons at site $i$. We denote by $\langle i, j\rangle_{\alpha}$ a nearest-neighbor bond in the $\alpha$ direction and $\tau^{\alpha}$ as the $\alpha$ th component of the vector of Pauli matrices $\tau:=\left(\tau^{1}, \tau^{2}, \tau^{3}\right)$ acting in spin space. The notations for nearest-neighbor $(\langle\cdot\rangle)$ and next-to-nearest-neighbor $(\langle\langle\cdot\rangle\rangle)$ pairs of sites are adopted in conventional form.

Following Ref. [23], we consider a half-filled band structure throughout and choose $t^{d}=1, t^{f}=-0.1, t^{d^{\prime}}=-0.4$, $t^{f^{\prime}}=0.04$, and $V=0.5$; all energy scales will be given in units of $t^{d}$. The form of Eq. (1) and the chosen parameters account for negligible interactions as well as bigger hopping amplitudes among the $d$ electrons as compared to the $f$ electrons. We chose the relative energy shift between $f$ - and $d$-orbitals $\epsilon_{f}$ and the interaction strength $U$ as free parameters as a function of which we will map out the phase diagram. We complement simplified slave-boson studies, which did not consider the possibility of magnetic order [23] or were performed at infinite interaction $U$ [24].

Slave-boson representation. To account for interactions, we apply the slave-boson representation of the operators $\widetilde{\boldsymbol{f}}_{i}^{(\dagger)}$, originally introduced by Kotliar and Ruckenstein [17], which has been generalized to be spin-rotation invariant (SRI) $[18,19]$ and to consider fluctuations around the PM saddle point [20,21]. We introduce the bosonic fields $e_{i}, d_{i}, p_{i, 0}$ and $\boldsymbol{p}_{i}:=\left(p_{i, 1}, p_{i, 2}, p_{i, 3}\right)$, labeling empty, doubly, and singly occupied states, respectively, i.e.,

$$
\begin{aligned}
& e_{i}^{\dagger}|\mathrm{vac}\rangle:=|\widetilde{\mathrm{vac}}\rangle, \quad d_{i}^{\dagger} f_{i, \uparrow}^{\dagger} f_{i, \downarrow}^{\dagger}|\mathrm{vac}\rangle:=\tilde{f}_{i, \uparrow}^{\dagger} \tilde{f}_{i, \downarrow}^{\dagger}|\widetilde{\mathrm{vac}}\rangle, \\
& \frac{1}{2} \sum_{\tilde{\alpha}=0}^{3} \sum_{\sigma^{\prime}}\left(p_{i, \tilde{\alpha}}^{\dagger} \tau^{\tilde{\alpha}}\right)_{\sigma \sigma^{\prime}} f_{i, \sigma^{\prime}}^{\dagger}|\mathrm{vac}\rangle:=\tilde{f}_{i, \sigma}^{\dagger}|\widetilde{\mathrm{vac}}\rangle,
\end{aligned}
$$

with $\mid$ vac $\rangle$ being the vacuum of the slave-boson representation containing a new set of auxiliary fermionic operators $\boldsymbol{f}_{i}^{\dagger}:=\left(f_{i, \uparrow}^{\dagger}, f_{i, \downarrow}^{\dagger}\right)$. The unity matrix is denoted by $\tau^{0}$. The Hilbert space defined by the slave particle operators has to be projected onto the physical Hilbert space by the application of constraints (see Supplemental Material [25]), which are inserted via Lagrange multiplier terms in the Lagrangian by introducing five new fields $i \alpha_{i}, i \beta_{i, 0}$, and $i \beta_{i, \alpha}$ per site $i$.

Mean-field approximation. We apply a mean-field ansatz, incorporating a static spin spiral with ordering vector $\boldsymbol{Q}$ of a possible magnetic ground state. Following Ref. [26], we replace the bosonic fields at the lattice site labeled by $i$ with lattice vector $\boldsymbol{r}_{i}$ by $b_{i} \rightarrow b$, where $b_{i}$ represents any of the fields $e_{i}, d_{i}, p_{0, i}, \alpha_{i}, \beta_{0, i}$, and define

$$
\boldsymbol{p}_{i} \rightarrow\left(\begin{array}{c}
\cos \left(\phi_{i}\right) \\
\sin \left(\phi_{i}\right) \\
0
\end{array}\right) p, \quad \text { and } \quad i \boldsymbol{\beta}_{i} \rightarrow\left(\begin{array}{c}
\cos \left(\phi_{i}\right) \\
\sin \left(\phi_{i}\right) \\
0
\end{array}\right) \beta .
$$

Here, we have $\phi_{i}=\boldsymbol{Q} \cdot \boldsymbol{r}_{i}, \beta \in \mathbb{R}$ and $b, p \in \mathbb{R}_{0}^{+}$. Within this mean-field ansatz, the free energy per site is given by [25]

$$
\begin{aligned}
\frac{F}{N}= & -\frac{T}{N} \sum_{\nu=1}^{8} \sum_{k} \ln \left[1+e^{-\epsilon_{k, Q}^{v} / T}\right]+U d^{2} \\
& +n \mu_{0}-\beta_{0}\left(2 d^{2}+p^{2}+p_{0}^{2}\right)-2 \beta p_{0} p,
\end{aligned}
$$

where $\epsilon_{\boldsymbol{k}, \boldsymbol{Q}}^{v}$ are the renormalized eigenvalues of the effective mean-field Hamiltonian, which implicitly depend on the slave-boson fields, and $T$ is the temperature. The index $v$ is a combined label for the spin, orbital, and sublattice degrees of freedom. The filling is fixed at $n=2$. As shown in Ref. [25], the free energy $F$ is invariant under global $\mathrm{SO}(3)$ rotations of $\boldsymbol{p}_{i}$.

We distinguish mean-field solutions with $p=0$, describing a PM state, and $p \neq 0$, signaling magnetic order. They are obtained by minimizing the free energy with respect to $d, p_{0}$, and $p$, while maximizing with respect to $\beta, \beta_{0}$, and $\mu_{0}$. Since there is a plethora of possible ordering vectors $\boldsymbol{Q}$ to consider, we first calculate the PM mean field by explicitly setting $p=$ $\beta=0$ and then perform a stability analysis of the saddle point by expanding the action $S$ up to second order in fluctuations of the bosonic fields.

Fluctuations around the paramagnetic saddle point. Within this expansion, the charge and spin susceptibility in momentum space are obtained as

$$
\begin{aligned}
\chi_{\mathrm{c}}(q) & :=\left\langle\delta n_{f}(-q) \delta n_{f}(q)\right\rangle, \\
\chi_{\mathrm{s}}^{\alpha \beta}(q) & :=\left\langle\delta S^{\alpha}(-q) \delta S^{\beta}(q)\right\rangle,
\end{aligned}
$$

where $S^{\alpha}=p_{0} p_{\alpha}$ represents the spin density operator projected to direction $\alpha$ and $n_{f}=2 d^{2}+p_{0}^{2}+\boldsymbol{p}^{2}$ is the charge density operator expressed in bosonic fields. We find $\chi_{\mathrm{s}}^{\alpha \beta}(q)$ to be proportional to the unit matrix and will hence refer to it as a scalar function $\chi_{\mathrm{s}}(q)$ [22]. Here, $q:=(\boldsymbol{q}, \omega)$ is the four-vector of the wave vector $\boldsymbol{q}$ and frequency $\omega$, and $\langle\cdot\rangle$ is the thermal expectation value of Gaussian fluctuations around the saddle point. We adopt the expressions for the susceptibilities from Refs. [22,27]. A sign change in the real part in the zero-frequency limit signals the onset of spontaneous charge or magnetic order. In the explored parameter domain, we do not find any charge instabilities. However, the real part of $\chi_{\mathrm{s}}$ exhibits a sign change in the two-dimensional parameter space 

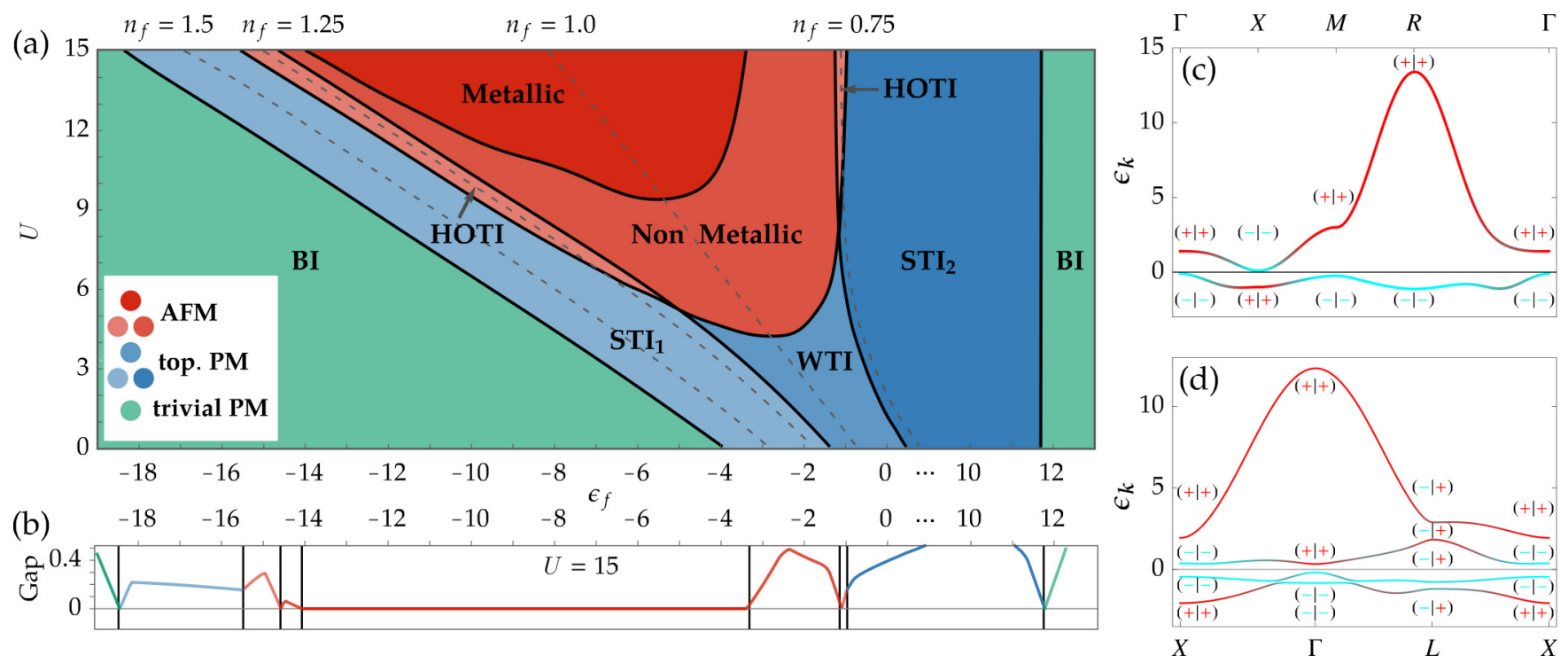

FIG. 1. (a) Topological phase diagram in the parameter space of $\left(\epsilon_{f}, U\right)$. BI, WTI, $\mathrm{STI}_{1}$, and $\mathrm{STI}_{2}$ phases are found in the PM region, whereas Non Metallic, Metallic, and HOTI phases appear in the AFM region. The phase boundaries are indicated by solid black lines. We further show trajectories of equal $f$-electron filling $n_{f}=1.5,1,25,1.0,0.75$ as dashed gray lines. (b) Gap plot as a function of $\epsilon_{f}$ for $U=15$ featuring topological gap closings as well as the metallic AFM region. (c) Bulk band structure for $\epsilon_{f}=-12$ and $U=10\left(\mathrm{STI}_{1}\right)$ on the high-symmetry path $\Gamma-X-M-R-\Gamma$ in the first BZ of the simple cubic lattice. (d) Bulk band structure for $\epsilon_{f}=-12$ and $U=12$ (HOTI, AFM) on the high-symmetry path $X-\Gamma-L-X$ in the first BZ of the face-centered-cubic lattice. Cyan (red) colored lines represent strong $f(c)$ occupation and $(+\mid-)$ the inversion eigenvalues of each Kramer's pair. Red band weights in the occupied spectrum of (c) and (d) indicate band inversion.

$\left(\epsilon_{f}, U\right)$, implying magnetic order. We compare the critical $U$ at vanishing $\omega$ for the instability with $\boldsymbol{q}=(\pi, \pi, \pi)$ to that of other ordering vectors. None of the latter led to an instability at smaller $U$, and thus we conclude that the magnetic ordering vector is $\boldsymbol{Q}=(\pi, \pi, \pi)$ (see Ref. [25]). The magnetic phase boundary can be seen in Fig. 1, containing a magnetic region approximately bound by the $f$-electron fillings $n_{f}=0.75$ and $n_{f}=1.25$ for interactions larger than $U=5$. The AFM mean-field spectrum allows for an insulatorto-metal transition by pushing noninteracting $d$ electrons to the Fermi level, which emerges at large $U$ around $n_{f}=1$. Investigating possible (incommensurate) magnetic or charge instabilities which could emerge on the AFM band structure deeper in the phase would require a susceptibility analysis of the magnetic band structure, which is beyond the scope of this Rapid Communication. A general form of the mean-field Hamiltonian for arbitrary ordering vector $\boldsymbol{Q}$ is derived in Ref. [25].

Topology of PM and AFM band structures. The solutions of the mean-field equations for $\boldsymbol{Q}=(\pi, \pi, \pi)$ yield magnetic and nonmagnetic domains in the parameter space of $\left(\epsilon_{f}, U\right)$. The resulting PM phase boundary coincides with the one obtained by analyzing the fluctuations around the paramagnetic saddle point, separating the phase diagram in a PM (green/blue) and AFM (red) domain in Fig. 1. The remaining phase boundaries indicate either a change of topology or a metal-to-insulator transition.

Using the renormalized band structure, we can study the band topology. In the PM case, the effective hopping Hamiltonian (see Ref. [25]) features antiunitary time reversal $\mathcal{T}:\left(\mathcal{T} c_{\sigma, \boldsymbol{k}}^{(\dagger)} \mathcal{T}^{-1}, \mathcal{T} f_{\sigma, \boldsymbol{k}}^{(\dagger)} \mathcal{T}^{-1}\right) \mapsto\left(\sigma c_{-\sigma,-\boldsymbol{k}}^{(\dagger)}, \sigma f_{-\sigma,-\boldsymbol{k}}^{(\dagger)}\right)$ and unitary inversion $\mathcal{I}:\left(\mathcal{I} c_{\sigma, k}^{(\dagger)} \mathcal{I}^{-1}, \mathcal{I} f_{\sigma, k}^{(\dagger)} \mathcal{I}^{-1}\right) \mapsto\left(c_{\sigma,-k}^{(\dagger)},-f_{\sigma,-k}^{(\dagger)}\right)$ symmetry, where $c_{\sigma,-k}^{(\dagger)}$ represents any fermionic operator with quantum numbers $(\sigma,-\boldsymbol{k})$. Because $(\mathcal{T} \mathcal{I})^{2}=-1$, bands are doubly degenerate at every $\boldsymbol{k}$. We can define the $\mathbb{Z}_{2}$-valued strong $\left(v_{0}^{\mathrm{PM}}\right)$ and weak $\left(v_{\alpha}^{\mathrm{PM}}\right)$ topological indices [23,28-30]:

$$
\begin{aligned}
& \text { strong: } \prod_{j=1}^{8} \prod_{n \in \text { occupied }} \xi\left[n, \Gamma_{j}\right]=(-1)^{\nu_{0}^{\mathrm{PM}}}, \\
& \text { weak: } \prod_{j ; k_{\alpha}=\pi} \prod_{n \in \text { occupied }} \xi\left[n, \Gamma_{j}\right]=(-1)^{\nu_{\alpha}^{\mathrm{PM}}} .
\end{aligned}
$$

Here, $\Gamma_{j}$ represents the time-reversal-invariant momenta (TRIM), which are $\Gamma^{\mathrm{PM}}=(0,0,0), \quad X^{\mathrm{PM}} \in\{(\pi, 0,0)$, $(0, \pi, 0),(0,0, \pi)\}, M^{\mathrm{PM}} \in\{(\pi, \pi, 0),(0, \pi, \pi),(\pi, 0, \pi)\}$, and $R^{\mathrm{PM}}=(\pi, \pi, \pi)$ in the first Brillouin zone (BZ) of the simple cubic lattice. $\xi\left[n, \Gamma_{j}\right]$ is the inversion eigenvalue of the $n$th Kramer's pair at the $\Gamma_{j}$ point. Due to the cubic symmetry in the PM phase, all the weak indices are equivalent, i.e., $v_{x}^{\mathrm{PM}}=v_{y}^{\mathrm{PM}}=v_{z}^{\mathrm{PM}}$. Therefore, we can maximally have four topologically distinct phases given by $\left(v_{0}^{\mathrm{PM}}, v_{\alpha}^{\mathrm{PM}}\right) \in\{(0,0),(0,1),(1,0),(1,1)\}$. We denote these phases by band insulator (BI), weak topological insulator (WTI) and strong topological insulator of type one and two $\left(\mathrm{STI}_{1}, \mathrm{STI}_{2}\right)$, respectively. We illustrate the factors of the strong index $v_{0}^{\mathrm{PM}}$ for the $\mathrm{STI}_{1}$ phase at $\left(\epsilon_{f}, U\right)=(-12,10)$ in Fig. 1(c): the eigenvalues $\xi\left[1, \Gamma_{j}\right]$ are $-1(+1)$ for the blue- (red)-colored portions of the energy bands, such that $\xi\left[1, \Gamma^{\mathrm{PM}}\right]=\xi\left[1, M^{\mathrm{PM}}\right]=\xi\left[1, R^{\mathrm{PM}}\right]=-1$, but $\xi\left[1, X^{\mathrm{PM}}\right]=1$ and, consequently, $\nu_{0}^{\mathrm{PM}}=1$.

In the magnetically ordered phases with ordering vector $Q=(\pi, \pi, \pi)$, the real-space primitive unit cell is doubled in size since each site $i$ is surrounded by six neighboring 
sites with opposite spin expectation value. In this AFM phase, the system has a pseudo-time-reversal symmetry $\mathcal{T}^{\prime}$ as the combination of $\mathcal{T}$ and the translation by $\hat{\boldsymbol{r}}_{\alpha}$ to a nearest neighbor in the $\alpha$ direction. Moreover, inversion symmetry $\mathcal{I}$ is retained. We find four doubly degenerate bands. The change in the unit cell from simple cubic to face-centered cubic results in eight new TRIMs, which are $\Gamma:=0, L:=$ $\boldsymbol{b}_{1} / 2, X:=\left(\boldsymbol{b}_{1}+\boldsymbol{b}_{2}\right) / 2$. Of these, $L$ and $X$ have, respectively, four and three partners obtained by $C_{4}$ rotations. Here, $\boldsymbol{b}_{1}:=$ $(-\pi, \pi, \pi), \boldsymbol{b}_{2}:=(\pi,-\pi, \pi)$, and $\boldsymbol{b}_{3}:=(\pi, \pi,-\pi)$ in units of $\left|\hat{r}_{\alpha}\right|^{-1}$ are the three primitive reciprocal lattice vectors of the fcc structure. Hence, the strong index,

$$
v^{\mathrm{AFM}}: \prod_{n \in \text { occupied }} \xi[n, \Gamma] \xi[n, X]=(-1)^{{ }^{\mathrm{AFM}},}
$$

corresponding to Eq. (7) remains $\mathbb{Z}_{2}$ valued, with the four $L$ points always yielding a trivial contribution. As shown in Fig. 1, each Kramer's pair at the $L$ point has two different inversion eigenvalues. Due to the translation operator in $\mathcal{T}^{\prime}$, which does not commute with the inversion operator at $L$, we find the relative phase between the eigenvalues of the two pairs to be $\exp \left(2 i \hat{\boldsymbol{r}}_{\alpha} L\right)=-1$. Thus, the weak index

$$
v_{\text {weak }}^{\mathrm{AFM}}: \prod_{n \in \text { occupied }}(\xi[n, X])^{2}=1=(-1)^{\nu_{\text {weak }}^{\mathrm{AFM}}}
$$

is always trivial. Therefore, apart from the metallic regime, we find a region with the trivial topological index $v^{\mathrm{AFM}}=0$, which is labeled Non Metallic, and a higher-order topological insulator (HOTI) $v^{\mathrm{AFM}}=1$, exhibiting topological states in two dimensions lower than the bulk [31-37]. We illustrate the factors of the index $v^{\mathrm{AFM}}$ for the HOTI phase at $\left(\epsilon_{f}, U\right)=$ $(-12,12)$ in Fig. 1(d).

The phase with nontrivial topology can further be classified as an axion insulator (AXI), which, depending on the orientation of the surfaces, can show gapless chiral hinge modes while the surface and bulk remain gapped. These modes are realized in a geometry that preserves $\mathcal{I}$ and breaks $\mathcal{T}^{\prime}$. Experimental evidence for such magnetically ordered topological materials was recently observed in $\mathrm{MnBi}_{2} \mathrm{Te}_{4}$ and $\mathrm{Bi}_{2} \mathrm{Se}_{3}$ thin films [38-40]. The hinge modes in a nanowire geometry are shown in Fig. 2. Surfaces that preserve $\mathcal{T}^{\prime}$ would feature a gapless Dirac cone.

Phase diagram. To sum up, depending on the parameter set $\left(\epsilon_{f}, U\right)$ that we probe, the effective fermionic part of Eq. (1) can realize a PM or an AFM phase with ordering vector $\boldsymbol{Q}=$ $(\pi, \pi, \pi)$. There are four topologically distinct subphases (BI, WTI, $\mathrm{STI}_{1}$, and $\mathrm{STI}_{2}$ ) in the PM phase, which are determined by the two topological indices $v_{0}^{\mathrm{PM}}$ and $v_{\alpha}^{\mathrm{PM}}$. On the other hand, there are three subphases (metallic, nonmetallic, and HOTI) in the AFM region. We find that the nonmetallic AFM phase features surface states, which are not topologically protected.

We further investigate the possibility of obtaining excitonic states with our formalism [25]. These charge-neutral collective modes have been suggested to account for the longstanding anomalies in $\mathrm{SmB}_{6}$ in several experimental observations and point to the relevance of excitons for the electronic structure of $\mathrm{SmB}_{6}$ [41,42]. However, we do not find any

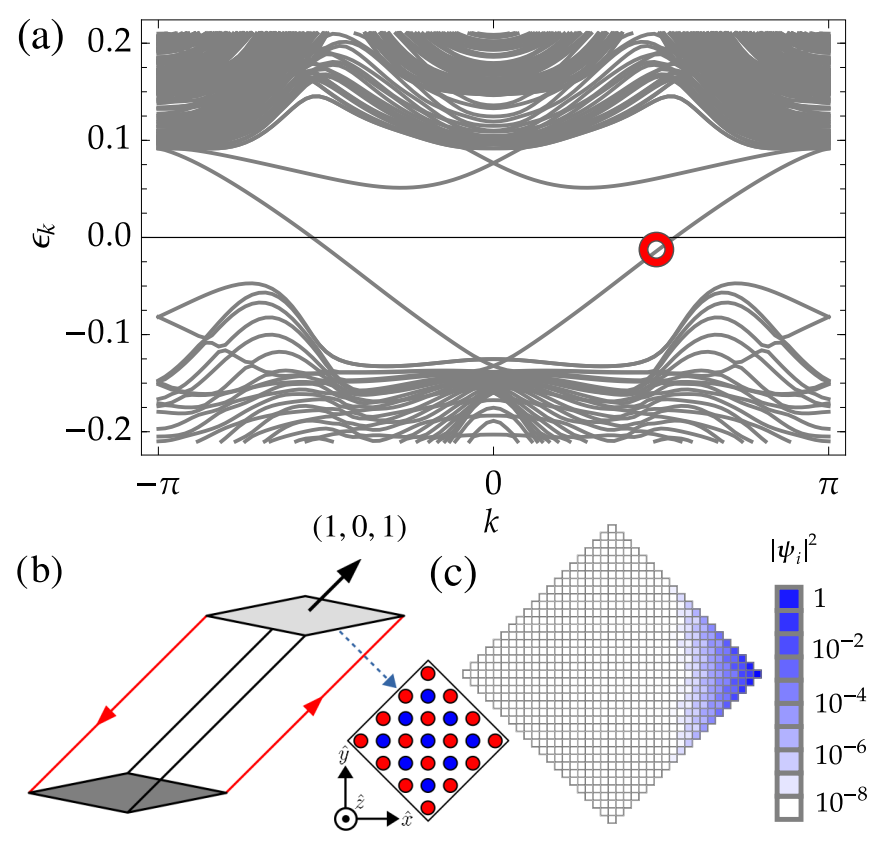

FIG. 2. (a) Band structure in the inclined column geometry depicted in (b) at $\left(\epsilon_{f}, U\right)=(-12,12)$ featuring chiral hinge modes. (b) An example of column geometry where the hinge mode appears. The AFM spin texture in each plane is shown in the diamondshaped inset. Red (blue) circles indicate spin $\downarrow$ ( $\uparrow$ ) in the fermionic part of the renormalized Hamiltonian. (c) Probability distribution in real space of the hinge state indicated in (a) by the red circle. $20 \times 20+19 \times 19=761$ sites are used for the plot.

evidence for excitonic states or bands separated from the band continuum within the dynamical spin susceptibility within our slave-boson treatment.

Conclusion. We studied the phase diagram of a paradigmatic model for three-dimensional TKIs using the scheme of Kotliar-Ruckenstein's slave-boson representation. To that end, we numerically implemented the analytical expressions of charge and spin susceptibility of a cubic and time-reversal symmetric system. We obtained a collection of phases in which topological properties and magnetic symmetry breaking are intertwined, yielding, among others, an axion insulator with chiral hinge modes.

Previous studies have theoretically predicted a bulk band inversion of $\mathrm{SmB}_{6}$ at the $X$ point and surface Dirac cones at the $\bar{X}$ and $\bar{\Gamma}$ points of the surface BZ $[23,43,44]$, which have been observed experimentally [45]. These characteristics coincide with the $\mathrm{STI}_{1}$ phase in our phase diagram. Further $\mathrm{SmB}_{6}$ shows strong indications of AFM ordering under pressure $[9,15]$, which is all captured in our minimal model within a small parameter range of $\left(\epsilon_{f}, U\right)$, corresponding to $f$-electron fillings $n_{f}$ between 1.5 and 1.25 . Judging from our results, we expect an indication of the sequence of phase transitions $\mathrm{STI}_{1}$ to HOTI to nonmetallic AFM in the specific heat as a function of pressure, which could be of interest to future experimental investigations.

Acknowledgments. The authors at the University of Zurich acknowledge support from the Swiss National Science Foundation (Grant No. 200021_169061) and from the European Unions Horizon 2020 research and innovation program 
(Grant No. ERC-StG-Neupert-757867-PARATOP). The work in Würzburg is funded by the Deutsche Forschungsgemeinschaft (DFG, German Research Foundation) through ProjectID No. 258499086-SFB 1170 and through the WürzburgDresden Cluster of Excellence on Complexity and Topology in Quantum Matter - ct.qmat Project-ID No. 39085490-EXC 2147. The authors also gratefully acknowledge the support of Markus Legner and Jannis Seufert.

The authors M.K., S.O., and D.R. contributed equally to this work.
[1] M. Dzero, K. Sun, V. Galitski, and P. Coleman, Phys. Rev. Lett. 104, 106408 (2010).

[2] M. Dzero, J. Xia, V. Galitski, and P. Coleman, Annu. Rev. Condens. Matter Phys. 7, 249 (2016).

[3] P. Nikolić, Phys. Rev. B 90, 235107 (2014).

[4] V. Alexandrov, P. Coleman, and O. Erten, Phys. Rev. Lett. 114, 177202 (2015).

[5] B. Roy, J. Hofmann, V. Stanev, J. D. Sau, and V. Galitski, Phys. Rev. B 92, 245431 (2015).

[6] Y. Nakajima, P. Syers, X. Wang, R. Wang, and J. Paglione, Nat. Phys. 12, 213 (2016).

[7] A. Thomson and S. Sachdev, Phys. Rev. B 93, 125103 (2016).

[8] K.-W. Chang and P.-J. Chen, Phys. Rev. B 97, 195145 (2018).

[9] Y. Zhou, Q. Wu, P. F. Rosa, R. Yu, J. Guo, W. Yi, S. Zhang, Z. Wang, H. Wang, S. Cai, K. Yang, A. Li, Z. Jiang, S. Zhang, X. Wei, Y. Huang, P. Sun, Y. feng Yang, Z. Fisk, Q. Si, Z. Zhao, and L. Sun, Sci. Bull. 62, 1439 (2017).

[10] I. Berman, N. Brandt, V. Moshchalkov, S. Pashkevich, V. Sidorov, E. Konovalova, and Y. Paderno, JETP Lett. 38, 477 (1983).

[11] J. Beille, M. B. Maple, J. Wittig, Z. Fisk, and L. E. DeLong, Phys. Rev. B 28, 7397(R) (1983).

[12] S. Gabáni, E. Bauer, M. Della Mea, K. Flachbart, Y. Paderno, V. Pavlík, and N. Shitsevalova, J. Magn. Magn. Mater. 272, 397 (2004)

[13] P. K. Biswas, Z. Salman, T. Neupert, E. Morenzoni, E. Pomjakushina, F. von Rohr, K. Conder, G. Balakrishnan, M. C. Hatnean, M. R. Lees, D. M. Paul, A. Schilling, C. Baines, H. Luetkens, R. Khasanov, and A. Amato, Phys. Rev. B 89, 161107(R) (2014).

[14] W. T. Fuhrman, J. Leiner, P. Nikolić, G. E. Granroth, M. B. Stone, M. D. Lumsden, L. DeBeer-Schmitt, P. A. Alekseev, J.-M. Mignot, S. M. Koohpayeh, P. Cottingham, W. A. Phelan, L. Schoop, T. M. McQueen, and C. Broholm, Phys. Rev. Lett. 114, 036401 (2015).

[15] A. Barla, J. Derr, J. P. Sanchez, B. Salce, G. Lapertot, B. P. Doyle, R. Rüffer, R. Lengsdorf, M. M. Abd-Elmeguid, and J. Flouquet, Phys. Rev. Lett. 94, 166401 (2005).

[16] P. Alekseev, V. Lazukov, K. Nemkovski, and I. Sadikov, J. Expt. Theor. Phys. 111, 285 (2010).

[17] G. Kotliar and A. E. Ruckenstein, Phys. Rev. Lett. 57, 1362 (1986).

[18] T. Li, P. Wölfle, and P. J. Hirschfeld, Phys. Rev. B 40, 6817 (1989).

[19] R. Frésard and P. Wölfle, Int. J. Mod. Phys. B 06, 685 (1992).

[20] T. Li, Y. S. Sun, and P. Wölfle, Z. Phys. B Condens. Matter 82, 369 (1991).

[21] W. Zimmermann, R. Frésard, and P. Wölfle, Phys. Rev. B 56, 10097 (1997).
[22] M. Klett, D. Riegler, T. Neupert, R. Thomale, and P. Wölfle (unpublished).

[23] M. Legner, A. Rüegg, and M. Sigrist, Phys. Rev. B 89, 085110 (2014).

[24] H. Li, Y. Zhong, Y. Liu, H.-G. Luo, and H.-F. Song, J. Phys.: Condens. Matter 30, 435601 (2018).

[25] See Supplemental Material at http://link.aps.org/supplemental/ 10.1103/PhysRevB.101.161112 for more details.

[26] B. Möller and P. Wölfle, Phys. Rev. B 48, 10320 (1993).

[27] D. Riegler, M. Klett, T. Neupert, R. Thomale, and P. Wölfle, arXiv:1912.07631.

[28] L. Fu and C. L. Kane, Phys. Rev. B 76, 045302 (2007).

[29] L. Fu, C. L. Kane, and E. J. Mele, Phys. Rev. Lett. 98, 106803 (2007).

[30] X.-L. Qi, T. L. Hughes, and S.-C. Zhang, Phys. Rev. B 78, 195424 (2008).

[31] A. M. Essin, J. E. Moore, and D. Vanderbilt, Phys. Rev. Lett. 102, 146805 (2009).

[32] R. S. K. Mong, A. M. Essin, and J. E. Moore, Phys. Rev. B 81, 245209 (2010).

[33] F. Schindler, A. M. Cook, M. G. Vergniory, Z. Wang, S. S. P. Parkin, B. A. Bernevig, and T. Neupert, Sci. Adv. 4, eaat0346 (2018).

[34] J. Ahn and B.-J. Yang, Phys. Rev. B 99, 235125 (2019).

[35] J. Langbehn, Y. Peng, L. Trifunovic, F. von Oppen, and P. W. Brouwer, Phys. Rev. Lett. 119, 246401 (2017).

[36] C. Yue, Y. Xu, Z. Song, H. Weng, Y.-M. Lu, C. Fang, and X. Dai, Nat. Phys. 15, 577 (2019).

[37] R.-X. Zhang, F. Wu, and S. D. Sarma, Phys. Rev. Lett. 124, 136407 (2020).

[38] M. M. Otrokov, I. I. Klimovskikh, H. Bentmann, D. Estyunin, A. Zeugner, Z. S. Aliev, S. Gaß, A. U. B. Wolter, A. V. Koroleva, A. M. Shikin et al., Nature (London) 576, 416 (2019).

[39] Y. Gong, J. Guo, J. Li, K. Zhu, M. Liao, X. Liu, Q. Zhang, L. Gu, L. Tang, X. Feng et al., Chin. Phys. Lett. 36, 076801 (2019).

[40] S.-Y. Xu, M. Neupane, C. Liu, D. Zhang, A. Richardella, L. A. Wray, N. Alidoust, M. Leandersson, T. Balasubramanian, J. Sánchez-Barriga et al., Nat. Phys. 8, 616 (2012).

[41] H. Ohta, R. Tanaka, M. Motokawa, S. Kunii, and T. Kasuya, J. Phys. Soc. Jpn. 60, 1361 (1991).

[42] J. Knolle and N. R. Cooper, Phys. Rev. Lett. 118, 096604 (2017).

[43] T. Takimoto, J. Phys. Soc. Jpn. 80, 123710 (2011).

[44] F. Lu, J. Z. Zhao, H. Weng, Z. Fang, and X. Dai, Phys. Rev. Lett. 110, 096401 (2013).

[45] N. Xu, P. Biswas, H. Dil, R. Dhaka, G. Landolt, S. Muff, C. Matt, Y. Wang, N. Plumb, M. Radovic, E. Pomjakushina, K. Conder, A. Amato, S. Borisenko, R. Yu, H. Weng, Z. Fang, X. Dai, J. Mesot, and M. Shi, Nat. Commun. 5, 4566 (2014). 